\title{
Review of modelling and remote control for excavators
}

\author{
Hongnian Yu*, Yang Liu, and Mohammad Shahidul Hasan \\ Faculty of Computing, Engineering and Technology, \\ Staffordshire University, Stafford, ST18 0AD UK \\ E-mail: h.yu@staffs.ac.uk \\ *Corresponding author
}

\begin{abstract}
An excavator is a typical hydraulic heavy-duty human-operated machine used in general versatile construction operations, such as digging, ground levelling, carrying loads, dumping loads, and straight traction. However, there are many tasks, such as hazard environment (nuclear decomposition, earthquake, etc) which is not suitable human working on site. The remotely controllable excavators are required to work in such environment. In this paper, we report the current progress of the on-going project. We investigate modelling and remote control issues of industry excavators. After reviewing the literature on the related work, architecture for remotely controllable excavators is proposed. The architecture covers actuators, modelling, sensors, image signal processing, communication networks, controllers, task \& path planning, human computer interaction, optimal design, co-simulation, and virtual training environment. The details of modelling, communication and control of a remotely controllable excavator are provided.
\end{abstract}

Keywords: Excavator, remote control, mechatronics, modelling.

Reference to this paper should be made as follows: Yu, H., Liu, Y. and Hasan, M. S. (2009) 'Review of modelling and remote control for excavators', Int. J. Advanced Mechatronic Systems, Vol. x, Nos. x/x/x, pp.xx-xx.

Biographical notes: Professor Hongnian Yu has held academic positions at the Universities of Yanshan, Sussex, Liverpool John Moor, Exeter, Bradford and Staffordshire. He is currently Professor of Computer Science and the head of the MCDS Research Group at Staffordshire University. He has extensive research experience in modelling and control of robots and mechatronics devices, and neural networks, mobile computing, modelling, scheduling, planning, and simulations of large discrete event dynamic systems, RFID with applications to manufacturing systems, supply chains, transportation networks and computer networks. He has published over 140 journal and conference research papers. He has held several research grants from EPSRC, the Royal Society, and the EU, AWM, as well as from industry. He is a member of EPSRC Peer Review College. He is a Program Chair of IEEE Conference on Networking, Sensing and Control in 2007, a General Chair of International conference on Software Knowledge Information Management and Applications in 2006, and is serving on various other conferences and academic societies. He was awarded the F.C. William Premium for his paper on adaptive and robust control of robot manipulators by the IEE Council in 1997.

Yang Liu received his B.Eng. degree in automation from Hunan University, China, in 2003 and M.Sc. degree in control systems from University of Sheffield, UK, in 2005. He is currently a Ph.D. student with the Faculty of Computing, Engineering, and Technology, Staffordshire University, UK. His research interests include control of underactuated mechanical systems, ground mobile robots, and robotics for medical applications.

Mohammad Shahidul Hasan received his BSc and first MSc in Computer Science from the University of Dhaka, Bangladesh. He obtained his second MSc in Computer and Network Engineering from Sheffield Hallam University, UK. He is currently pursuing his $\mathrm{PhD}$ at Staffordshire University, UK in Networked Control Systems over Mobile Ad-hoc Network (MANET). He has worked as full time lecturer of computer science and engineering in Bangladesh and is engaged in part time teaching at Staffordshire University. His research 
interests include computer networks, networked control systems, remotely controllable mobile robot systems etc.

\section{INTRODUCTION}

Earthmoving machines, such as bulldozers, wheel loaders, excavators, scrapers, and graders are common in construction. An excavator is a typical hydraulic heavy-duty human-operated machine used in general versatile construction operations, such as digging, ground levelling, carrying loads, dumping loads, and straight traction. However, there are many tasks, such as hazard environment (nuclear decomposition, earthquake, etc) which is not suitable for human to work on site. The remotely controllable excavators are required to work in such environment. We will make a brief review from the two aspects: 1) modelling of excavators and 2) remote control of excavators.

On the modelling aspect, early research work on the dynamic model of excavators has been done by Vaha et al. (1993). Based on Vaha et al. (1993), Koivo et al. (1996) did further studies on the modelling of excavator dynamics during digging operations. Later on, a number of researchers investigated the feasibility of autonomous excavation. Many of these studies have addressed the possible use of an autonomous excavator (Le et al., 1998; Bradley and Seward, 1998).

Based on the earlier research work, implementation of an autonomous teleoperated excavator mainly focused on three parts: modelling, parameter identification, and control strategy. The key reason for modelling and parameter identification during the digging operation is to provide online parameters for the development of an autonomous strategy. In Tafazoli et al. (1999), an experimental determination approach of the link parameters and friction coefficients was developed on the excavator arm. Zweiri et al. (2004) presented another robust, fast, and simple technique for the experimental identification of the link parameters and friction coefficients of a full-scale excavator arm. Furthermore, in order to carry out autonomous excavation, an online soil parameter estimation scheme was proposed by Tan et al. (2005). At the earlier stage of study on excavation, impedance control was considered as a popular robust control approach to achieve compliant motion in contact tasks. Details of robust impedance control for a hydraulic excavator have been presented by Lu et al. (1995) and Ha et al. (2000). In Tafazoli et al. (2002), a position-based impedance controller was presented on various contact experiments by using an instrumented mini-excavator. Rather than excavation control strategy, motion and path planning for autonomous excavation have also been studied in a number of research papers by Bernold (1993) and Singh (1995). In Saeedi et al. (2005), a vision-based control system for a tracked excavator was presented. The system includes several controllers that collaborate to move the excavator from a starting position to a goal position. In the paper, both path-tracking accuracy and slippage control problems have been addressed.

The idea of teleoperated excavator was studied by Parker et al. (1993), Lawrence et al. (1995), and Kim et al. (2008) based on the force-feedback control. In a teleoperated excavator system, if the operator cannot sense the condition of contact, the work efficiency will decrease compared to a direct control by the human operator. So, design of the joystick with proper force feedback can make skilful operators adapt their operation to the excavating environment based on their empirical knowledge, and can realise efficient excavation. In Lawrence et al. (1995), it has proposed the single joystick endpoint velocity control, which is controlling joystick stiffness as a function of endpoint force. It was found to be both a stable and effective form of feedback for a system where joystick position maps to endpoint velocity. Different from controlling a real hydraulic excavator, there are many studies which implement their work on the virtual excavator including development and evaluation of the controller (Dimaio et al., 1998), operator training (Tao et al., 2008), and investigation of remote control issues (Yang et al., 2008). Apparently, the virtual excavator system is a low-cost, safe, and reliable system that can both test the system and the control strategy in virtual environment.

As discussed above, many research studies have focused on modelling and controller development stages, but few literature studies the remote operation from a network communication point of view. Furthermore, it is found that efficiency of excavation by human operator (Sakaida et al., 2008) is a notable issue that has potential commercial value. On the other hand, a teleoperated excavator has always been desired by industry and manufacturing during the past two decades. Much of the work on terrestrial excavation has focused on teleoperation, rather than on the system requirements for autonomous operation (Ha et al., 2002). However, although remarkable and valuable progress has been made on automated excavation, teleoperation of a full-scale excavator has not been commercially demonstrated.

This paper identifies the issues on designing a remotely controllable excavator. Section 2 identifies the requirements of remotely controllable excavators and proposes remote control architecture of excavators. Section 3 provides the forward kinematics, inverse kinematics and dynamics of excavators. Those models will provide the basis for the system design, development of the controllers, task/path planning, simulation, validation etc. Section 4 presents several control schemes for controlling excavators. Some of those control schemes are based on the authors' previous work conducted in robotics context. Section 5 proposes a wireless networked control scheme for excavators. Finally the conclusions and future works are given in section 6 . 


\section{REQUIREMENTS OF REMOTELY CONTROLLABLE EXCAVATORS}

Remotely controllable robots or excavators using wired networks restrict the coverage area and offer very limited flexibility. On the other hand, wirelessly controlled mobile robots or excavators provide the freedom from wired networks and support a higher degree of movement and hence are preferable to wired versions. Researchers and many industries are concentrating more and more on such systems as they are suitable for various applications e.g. nuclear plant decommissioning, disaster rescue, military operation etc. The proposed overall system is shown in Figure 1. The excavator is equipped with the necessary sensors and camera for gathering data (signal), actuators for moving it and a wireless communication module to transmit the signals, etc. The sensor and camera data are transmitted to the control (decision making) centre through the wireless network which is composed of multiple mobile robots. The primary responsibility of these robots is to relay the data to and from the excavator. These robot nodes can also have a camera mounted on them to provide additional visual feedback of the excavator to the control centre. The sensor and camera data are monitored and analysed at the control and decision making centre to make the right decision and to send the necessary action or command to the excavator over the wireless network.

Staffordshire University with a UK based excavator developer presented a physical demonstration in an exhibition hosted by a UK based nuclear decommissioning company, where ten universities and fourteen companies in robotics made the presentations. The demonstration system made by the Staffordshire University team involved a dummy excavator, an observer robot being controlled over a Mobile Ad-hoc Network (MANET). This paper presents the system design for a remotely controllable excavator based on the experience and the requirements for such applications.

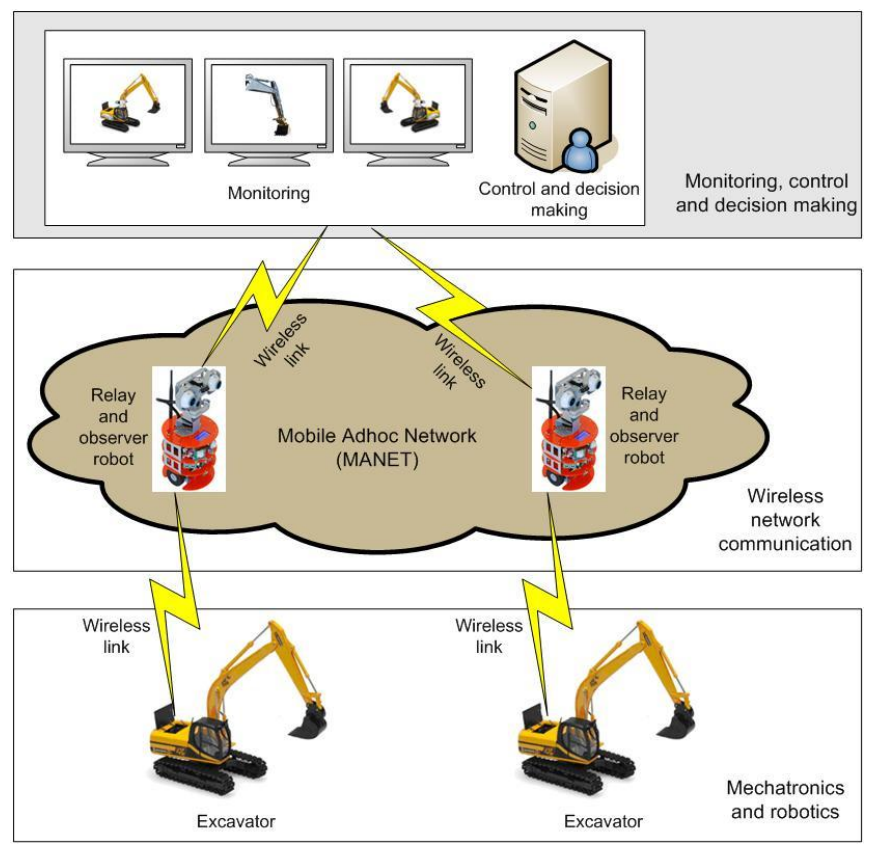

Figure 1: The overall system design.

In order to develop the remotely controllable autonomous excavators, the following issues and requirements should be investigated.

\subsection{Modeling of Excavators}

During the digging operation, it will require not only the bucket trajectory but also the forces exerted by the bucket on the soil. Therefore, the modelling of the excavator will involve (Koivo et al., 1994 and 1996):

1) the kinematics which give the trajectory of the excavator bucket based on the trajectory of the excavator arm joints,

2) the inverse kinematics which give the desired joint variables corresponding to the desired bucket trajectory,

3) the dynamics which describe the behaviour of the excavator system,

4) modelling of the interaction between the excavator bucket and the environment which is necessary for the remote control during the digging task.

\subsection{Sensors and Camera}

Remote or autonomous controls for the excavators can potentially improve the operational safety and efficiency. Sensors are crucial to this requirement, since feedback signals are necessary to carry out an unmanned or indirect controlled task. The sensors used in remote control will include position / velocity sensors that monitor the joint angles/velocities, force sensors that detect the interactive force between the excavator bucket and the environment, and the vibration sensors that measure the vibration status of excavators. In addition, the camera is another key sensor which can be used for the vision-based control system (Saeedi et al., 2000 and 2005). 
From the vision information, the operator can better operate the excavator remotely.

\subsection{Actuators}

There are a number of nonlinearities affecting the dynamics of hydraulic actuators, such as the basic flow equation through an orifice, flow forces on valve spools, and friction (Tafazoli, 1997). To overcome these nonlinear effects, investigation of the hydraulic actuator is necessary (Tafazoli, et al., 2002).

\subsection{Communication systems between excavators and remote controllers (decision-making)}

With the development of high-speed networks capable of carrying real-time traffic and a network interface with built-in sensor/actuator, control systems over network have become an interesting area of research (Cervin, 2003), (Cervin et al., 2002). Nowadays a low cost and easily deployable remotely controllable excavator system can be implemented using IEEE 802.11 standards as shown in Figure 2.

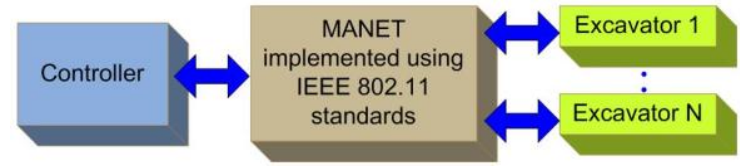

Figure 2: Block diagram of remotely controlled excavator systems.

\subsection{Signal and Image signal processing}

The data (signal) measured from the sensors and cameras will be unavoidably contaminated by all sort of noise. To extract the required valid signal and data for the purposes of control and 2D/3D virtual view, the certain type of filters and data processing meads are needed (Fua, 1993; Schmid and Bauckhage, 1998). The data captured by multiple cameras mounted on the excavator and the observer robots shown in Figure 3 can be processed and combined to produce a complete 3D virtual view of the excavator surroundings (Shapiro, et al., 1995). However, this process will consume valuable wireless network bandwidth to transfer video stream and involves heavy computation of image processing.

\subsection{Intelligent control}

To achieve the goal of remote control, adaptive and robust control law is required to compensate for the nonlinear dynamics of the excavator system. For example, Vossoughi and Salcudean (2000) used the feedback linearisation technique, and in Heinrichs et al. (1997), a nonlinear proportional-integral controller was used. An impedance controller was used by Tafazoli, et al. (2002) on a teleoperated excavator. In addition, excavators often conduct respective tasks; therefore iterative control approaches can be applied.

\subsection{Path planning and task planning}

Remote control of the excavator in natural environments requires planning every movement in order to avoid any obstacle and to locate the machine at each time with respect to a global coordinate system (Saeedi, et al., 2005). With the application of an effective path planning, human steering of the excavator can be removed. Task panning (Singh, 1995) is to design an operation sequence based on the tasks to be done.
Human operative error can be minimised or completely removed, and more consistent operation of the machine can be achieved to increase efficiency.

\subsection{Human computer interaction}

The system can have two modes of operation: manual and autonomous. In manual mode, an operator can observe different views i.e. excavator and observer robot views on screen and move the excavator manually using a joystick (Kim et al., 2008; Yang et al., 2008) attached to the control centre computer. In the autonomous mode, the intelligent control centre can move the excavator autonomously based on the sensor and camera data.

\subsection{Optimum overall design}

The overall design of the excavator is shown in Figure 3. It is equipped with an adjustable overhead camera, IEEE 802.11 wireless communication module and several electromagnetic and ultrasonic sensors around it. The overhead camera produces the operator's view (Saeedi, et al., 2005). Two mobile observer robots carrying a remotely adjustable camera on both sides of the excavator will provide the left and right views at the controller end.

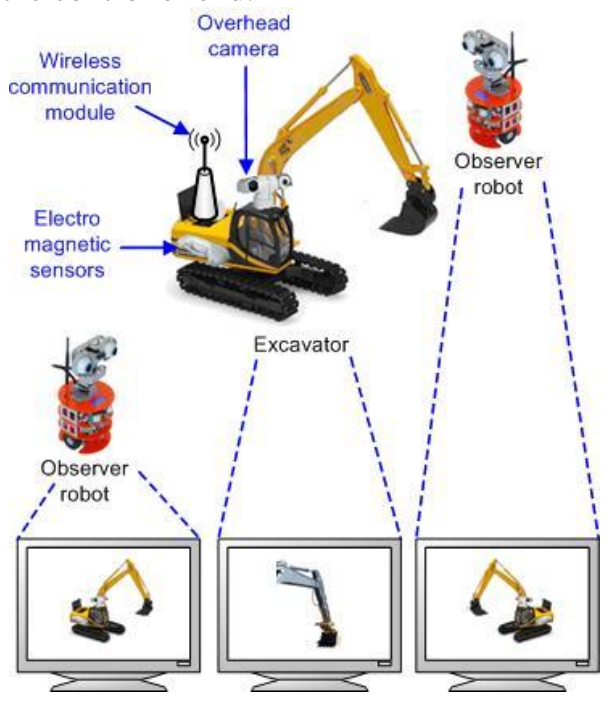

Figure 3: The overall excavator design.

\subsection{Simulation environment: Co-simulation}

This paper adopts the co-simulation framework developed in (Hasan et al., 2009) utilising MATLAB-SIMULINK to model the plant-controller and OPNET to simulate the network to accelerate the remotely controllable excavator system research by producing more realistic simulation results.

\subsection{Virtual training environment}

Providing training for new operators on actual systems can be expensive in terms of time and money. A virtual training environment (Dimaio et al., 1998) shown in Figure 4 can reduce the cost dramatically. The trainee operator interacts with the system through the joysticks. The excavator's dynamics are simulated on a computer (Makkonen, et al., 2006). 


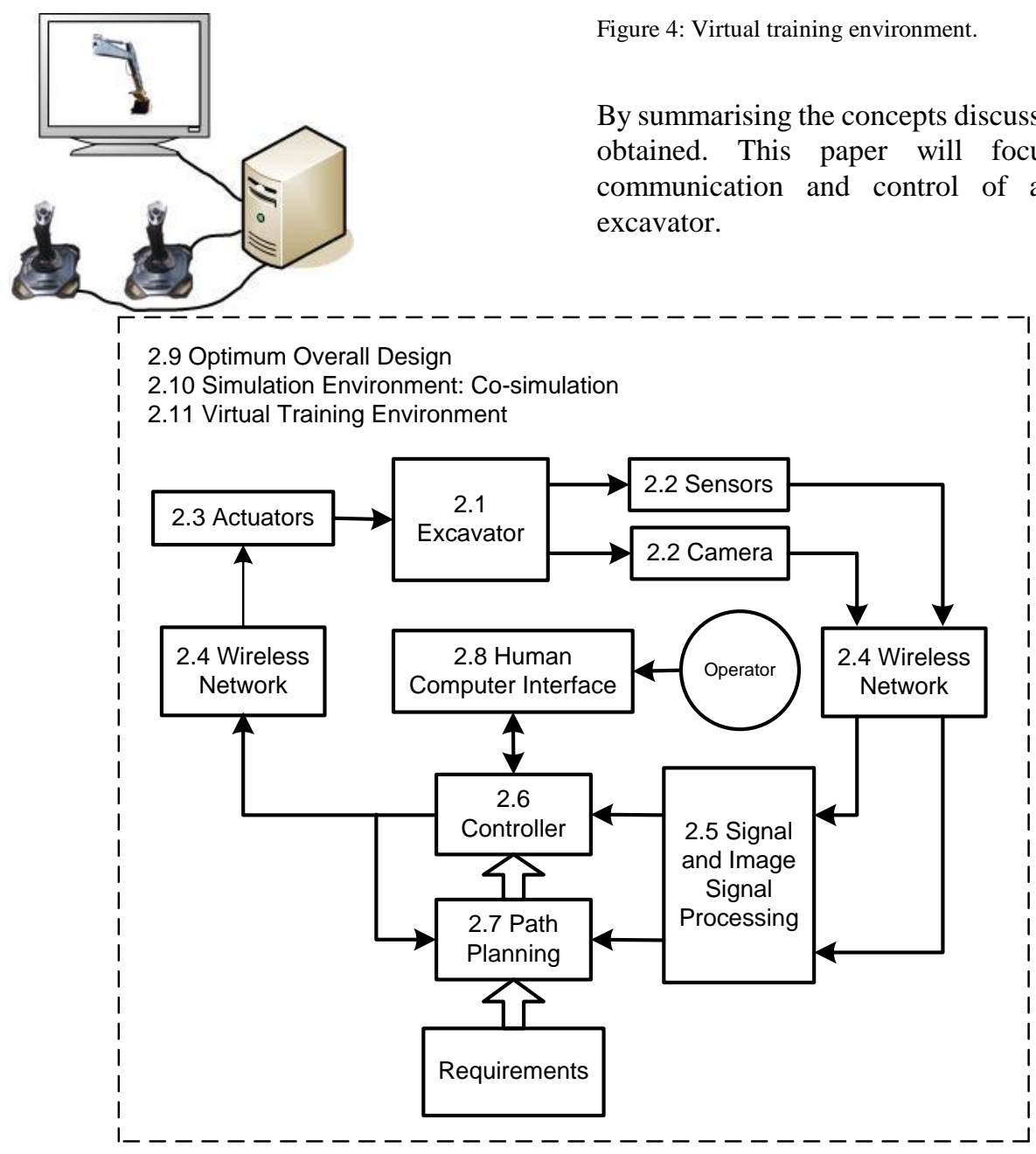

Figure 5: Remote control architecture of excavators.

\section{MODELLING OF EXCAVATORS}

\subsection{Kinematics}

The excavator schematic diagram is shown in Figure 6. The coordinate systems are assigned systematically by applying the Denavit-Hartenberg convention in Koivo (1994). To describe the positions of the points on the excavator, the Cartesian coordinate systems are defined to attach to the links, which include a fixed Cartesian coordinate system with the origin on the body of the excavator. It is noticed that the rotational axis for the first link (i.e. the base) is vertical, whereas the rotational axes for the other links are horizontal.

The forward kinematics is used to describe the positions and orientations of the points on the excavator in the Cartesian coordinate for the given joint positions during the digging operation. The problem can be summarised as below:

For the given $\Theta=\left[\begin{array}{lll}\theta_{2} & \theta_{3} & \theta_{4}\end{array}\right]^{\mathrm{T}}$, find the coordinate $\mathrm{P}=[\mathrm{X}$ Y $\mathrm{Z}]^{\mathrm{T}}=\left[\mathrm{f}_{\mathrm{x}}(\Theta) \mathrm{f}_{\mathrm{y}}(\Theta) \mathrm{f}_{\mathrm{z}}(\Theta)\right]^{\mathrm{T}}$. 


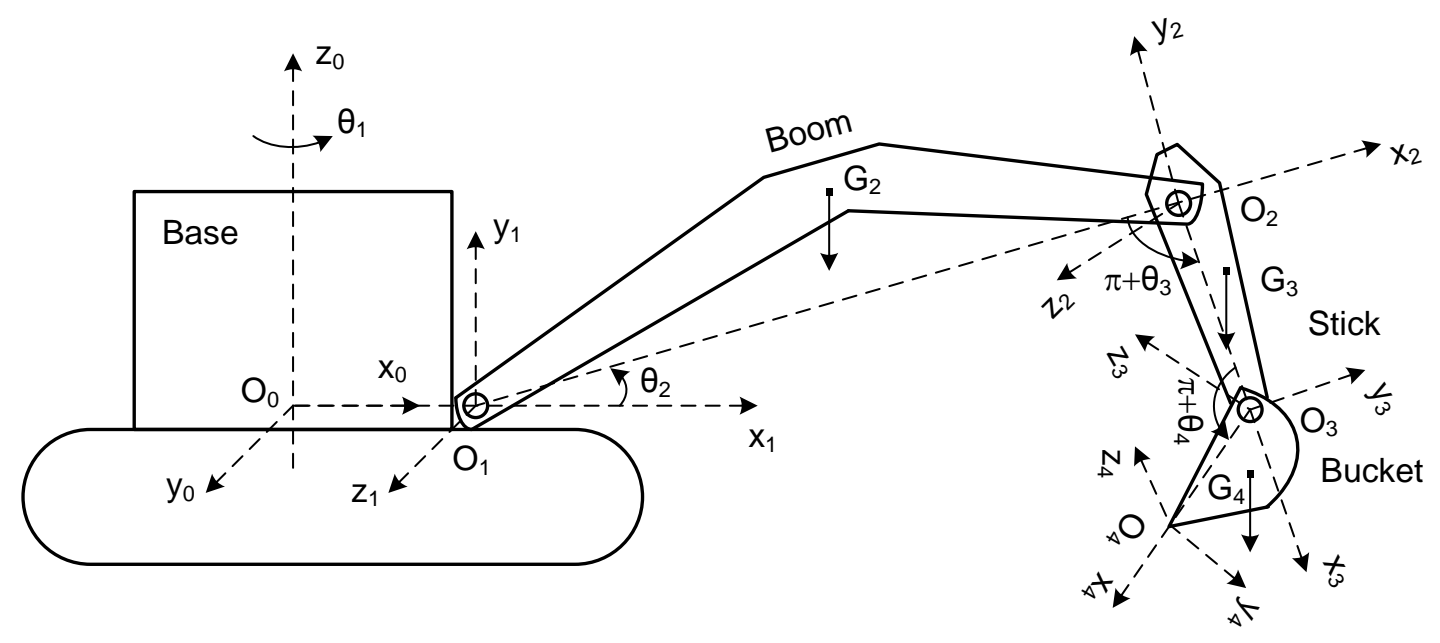

Figure 6: Schematic diagram of the excavator

To determine the positions of the points on the excavator in the base Cartesian coordinate frame, the relations between the fixed coordinate system and other coordinate systems is necessary. Therefore, the transformation matrix relating two adjacent coordinate frames was studied by Koivo et al. (1996) as follows:

$$
A_{i-1}^{i}=\left[\begin{array}{cccc}
\cos \theta_{i} & -\cos \alpha_{i} \sin \theta_{i} & \sin \alpha_{i} \sin \theta_{i} & a_{i} \cos \theta_{i} \\
\sin \theta_{i} & \cos \alpha_{i} \cos \theta_{i} & -\sin \alpha_{i} \cos \theta_{i} & a_{i} \sin \theta_{i} \\
0 & \sin \theta_{i} & \cos \alpha_{i} & d_{i} \\
0 & 0 & 0 & 1
\end{array}\right]
$$

where $\alpha_{\mathrm{i}}$ is the twist angle of link $\mathrm{i}, \mathrm{a}_{\mathrm{i}}$ is the length of link $\mathrm{i}$, and $\mathrm{d}_{\mathrm{i}}$ is the offset distance in link $\mathrm{i}, \mathrm{i}=1,2,3$, and 4 .

By given the coordinates of the origin in each coordinate frame $\mathrm{O}_{\mathrm{i}}$, the coordinates of points $\mathrm{O}_{\mathrm{i}}$ in the base coordinate frame can be described as follows using the matrix (1):

$$
P_{0}^{O_{i}}=A_{0}^{i} P_{i}^{O_{i}}
$$

where vector $P_{i}^{O_{i}}=\left[\begin{array}{llll}0 & 0 & 0 & 1\end{array}\right]^{T}$ specifies point $\mathrm{O}_{\mathrm{i}}$ in the $\mathrm{i}^{\text {th }}$ coordinate frame. So, by using equation (2), we can describe the origin of each coordinate frame $\mathrm{O}_{\mathrm{i}}$ in the base coordinate frame as follows:

$$
\begin{aligned}
& P_{0}^{O_{1}}=A_{0}^{1} P_{1}^{O_{1}}=\left[\begin{array}{llll}
a_{1} c_{1} & a_{1} s_{1} & 0 & 1
\end{array}\right]^{T} \\
& P_{0}^{O_{2}}=A_{0}^{1} A_{1}^{2} P_{2}^{O_{2}} \\
& =\left[\begin{array}{llll}
a_{2} c_{1} c_{2}+a_{1} c_{1} & a_{2} c_{2} s_{1}+a_{1} s_{1} & a_{2} s_{2} & 1
\end{array}\right]^{T} \\
& P_{0}^{O_{3}}=A_{0}^{1} A_{1}^{2} A_{2}^{3} P_{3}^{O_{3}}=\left[\begin{array}{c}
c_{1}\left(a_{3} c_{23}+a_{2} c_{2}+a_{1}\right) \\
s_{1}\left(a_{3} c_{23}+a_{2} c_{2}+a_{1}\right) \\
a_{3} s_{23}+a_{2} s_{2} \\
1
\end{array}\right] \\
& P_{0}^{O_{4}}=A_{0}^{1} A_{1}^{2} A_{2}^{3} A_{3}^{4} P_{4}^{O_{4}} \\
& =\left[\begin{array}{c}
c_{1}\left(a_{4} c_{234}+a_{3} c_{23}+a_{2} c_{2}+a_{1}\right) \\
s_{1}\left(a_{4} c_{234}+a_{3} c_{23}+a_{2} c_{2}+a_{1}\right) \\
a_{4} s_{234}+a_{3} s_{23}+a_{2} s_{2} \\
1
\end{array}\right]
\end{aligned}
$$

where $\mathrm{c}_{\mathrm{i}}=\cos \theta_{\mathrm{i}}, \mathrm{s}_{\mathrm{i}}=\sin \theta_{\mathrm{i}}, \theta_{23}=\theta_{2}+\theta_{3}$, and $\theta_{234}=\theta_{2}+\theta_{3}+\theta_{4}$.

\subsection{Inverse kinematics}

The inverse kinematics (or backward kinematic relations) is used to determine the joint positions for the given desired coordinate points in the Cartesian coordinate. The problem of inverse kinematics can be summarised as below:

For the given $P=[X \quad Y Z]^{T}$, find the joint angles $\Theta=\left[\begin{array}{ll}\theta_{2} & \theta_{3}\end{array}\right.$ $\left.\theta_{4}\right]^{\mathrm{T}}=\left[\mathrm{g}_{2}(\mathrm{P}) \mathrm{g}_{3}(\mathrm{P}) \mathrm{g}_{4}(\mathrm{P})\right]^{\mathrm{T}}=\left[\mathrm{f}_{\mathrm{x}}^{-1}(\Theta) \mathrm{f}_{\mathrm{y}}^{-1}(\Theta) \mathrm{f}_{\mathrm{z}}^{-1}(\Theta)\right]^{\mathrm{T}}$

According to Tafazoli (1997), the inverse kinematic model of the excavator is given as follow:

$$
\begin{aligned}
& \theta_{1}=\theta_{b} \\
& l_{1}=\sqrt{\left(r_{b}-a_{1}\right)^{2}+z_{b}^{2}} \\
& \xi_{1}=a \tan \left[z_{b} /\left(r_{b}-a_{1}\right)\right] \\
& l=\sqrt{l_{1}^{2}+a_{4}^{2}-2 l_{1} a_{4} \cos \left(\xi_{1}-\alpha\right)} \\
& \xi_{2}=a \sin \left[\left(z_{b}-a_{4} \sin \alpha\right) / l\right] \\
& \theta_{2}=\xi_{2}+a \cos \left[\left(a_{2}^{2}+l^{2}-a_{3}^{2}\right) /\left(2 a_{2} l\right)\right] \\
& \theta_{3}=-\pi+a \cos \left[\left(a_{2}^{2}+a_{3}^{2}-l^{2}\right) /\left(2 a_{2} a_{3}\right)\right] \\
& \theta_{4}=\alpha-\theta_{23}
\end{aligned}
$$

\subsection{Dynamics}

The dynamic model will describe the relationships among the joint angles. The dynamic model of the excavator can be expressed concisely using the form of the well-known rigid-link manipulator equations of motion (Yu, 1998):

$$
D(\theta) \ddot{\theta}+C(\theta, \dot{\theta}) \dot{\theta}+G(\theta)+B(\dot{\theta})=\Gamma \tau-\tau_{L}
$$

where $\theta=\left[\begin{array}{llll}\theta_{1} & \theta_{2} & \theta_{3} & \theta_{4}\end{array}\right]^{T}$ is the vector of measured joint angles as defined in Figure 6; $\mathrm{D}(\theta)$ represents inertia; $C(\theta, \dot{\theta})$ represents Coriolis and centripetal effects; $G(\theta)$ represent gravity forces; $B(\dot{\theta})$ represent frictions; $\Gamma$ is the

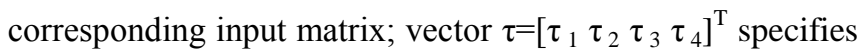
the torques acting on the joint shafts; $\tau_{\mathrm{L}}$ represents the interactive torques between the bucket and the environment during the digging operation. 
According to Koivo et al. (1996), $\mathrm{D}(\theta), C(\theta, \dot{\theta}), \mathrm{G}(\theta), \Gamma(\theta)$, and $\mathrm{F}_{\mathrm{L}}$ are given by the following expression:

$$
D(\theta)=\left[\begin{array}{llll}
D_{11} & D_{12} & D_{13} & D_{14} \\
D_{21} & D_{22} & D_{23} & D_{24} \\
D_{31} & D_{32} & D_{33} & D_{34} \\
D_{41} & D_{42} & D_{43} & D_{44}
\end{array}\right]
$$

where

$$
\begin{aligned}
& \begin{array}{l}
D_{44}=I_{b u}+M_{b u} r_{4}^{2} \\
D_{33}=D_{44}+I_{s t}+M_{s t} r_{3}^{2}+M_{b u}\left[a_{3}^{2}+2 a_{3} r_{4} \cos \left(\theta_{4}+\alpha_{4}\right)\right] \\
D_{22}=D_{33}+I_{b o}+M_{b o} r_{2}^{2}+M_{s t}\left[a_{2}^{2}+2 a_{2} r_{3} \cos \left(\theta_{3}+\alpha_{3}\right)\right] \\
+M_{b u}\left[a_{2}^{2}+2 a_{2} a_{3} c_{3}+2 a_{2} r_{4} \cos \left(\theta_{34}+\alpha_{4}\right)\right] \\
D_{34}=D_{44}+M_{b u} a_{3} r_{4} \cos \left(\theta_{4}+\alpha_{4}\right) \\
D_{24}=D_{34}+M_{b u} a_{2} r_{4} \cos \left(\theta_{34}+\alpha_{4}\right) \\
D_{23}=D_{24}+I_{s t}+M_{s t}\left[r_{3}^{2}+a_{2} r_{3} \cos \left(\theta_{3}+\alpha_{3}\right)\right] \\
+M_{b u}\left[a_{3}^{2}+a_{2} a_{3} c_{3}+a_{3} r_{4} \cos \left(\theta_{4}+\alpha_{4}\right)\right]
\end{array} \\
& \qquad(\theta, \dot{\theta})=\left[\begin{array}{llll}
C_{11} & C_{12} & C_{13} & C_{14} \\
C_{21} & C_{22} & C_{23} & C_{24} \\
C_{31} & C_{32} & C_{33} & C_{34} \\
C_{41} & C_{42} & C_{43} & C_{44}
\end{array}\right]
\end{aligned}
$$

where

$$
\begin{aligned}
& C_{22}=-M_{s t} a_{2} r_{3} \dot{\theta}_{23} \sin \left(\theta_{3}+\alpha_{3}\right)-M_{b u} a_{2} a_{3} \dot{\theta}_{23} s_{3} \\
&-M_{b u} a_{2} r_{4} \dot{\theta}_{234} \sin \left(\theta_{34}+\alpha_{4}\right) \\
& C_{23}=-M_{s t} a_{2} r_{3} \dot{\theta}_{23} \sin \left(\theta_{3}+\alpha_{3}\right)-M_{b u} a_{2} a_{3} \dot{\theta}_{23} s_{3} \\
&-M_{b u} a_{2} r_{4} \dot{\theta}_{234} \sin \left(\theta_{34}+\alpha_{4}\right) \\
& C_{24}=-M_{b u} a_{2} r_{4} \dot{\theta}_{234} \sin \left(\theta_{34}+\alpha_{4}\right) \\
& C_{32}= a_{2} \dot{\theta}_{2}\left[M_{b u} a_{3} s_{3}+M_{s t} r_{3} \sin \left(\theta_{3}+\alpha_{3}\right)\right] \\
&-M_{b u} a_{3} r_{4} \dot{\theta}_{234} \sin \left(\theta_{4}+\alpha_{4}\right) \\
& C_{33}=-M_{b u} a_{3} r_{4} \dot{\theta}_{234} \sin \left(\theta_{4}+\alpha_{4}\right) \\
& C_{34}=-M_{b u} a_{3} r_{4} \dot{\theta}_{234} \sin \left(\theta_{4}+\alpha_{4}\right) \\
& C_{42}= M_{b u} r_{4} \dot{\theta}_{2}\left[a_{2} \sin \left(\theta_{34}+\alpha_{4}\right)+a_{3} \sin \left(\theta_{4}+\alpha_{4}\right)\right] \\
&+M_{b u} a_{3} r_{4} \dot{\theta}_{3} \sin \left(\theta_{4}+\alpha_{4}\right) \\
& C_{43}= M_{b u} a_{3} r_{4} \sin \left(\theta_{4}+\alpha_{4}\right) \\
& C_{44}= 0 \\
& G(\theta)=\left[\begin{array}{llll}
G_{1} & G_{2} & G_{3} & G_{4}
\end{array}\right]^{T}
\end{aligned}
$$

where

$$
\begin{aligned}
& G_{2}=\left(M_{b u}+M_{s t}\right) g a_{2} c_{2}+M_{b o} g r_{2} \cos \left(\theta_{2}+\alpha_{2}\right) \\
& G_{3}=M_{b u} g a_{3} c_{23}+M_{s t} g r_{3} \cos \left(\theta_{23}+\alpha_{3}\right) \\
& G_{4}=M_{b u} g r_{4} \cos \left(\theta_{234}+\alpha_{4}\right) \\
& B(\dot{\theta})=\left[\begin{array}{llll}
B_{b a} \dot{\theta}_{1} & B_{b o} \dot{\theta}_{2} & B_{s t} \dot{\theta}_{3} & B_{b u} \dot{\theta}_{4}
\end{array}\right]^{T} \\
& \Gamma=\left[\begin{array}{cccc}
\Gamma_{11} & \Gamma_{12} & \Gamma_{13} & \Gamma_{14} \\
\Gamma_{21} & 1 & -1 & 0 \\
\Gamma_{31} & 0 & 1 & -1 \\
\Gamma_{41} & 0 & 0 & 1
\end{array}\right]
\end{aligned}
$$

The interaction between the excavator bucket and the environment is presented in Figure 7.

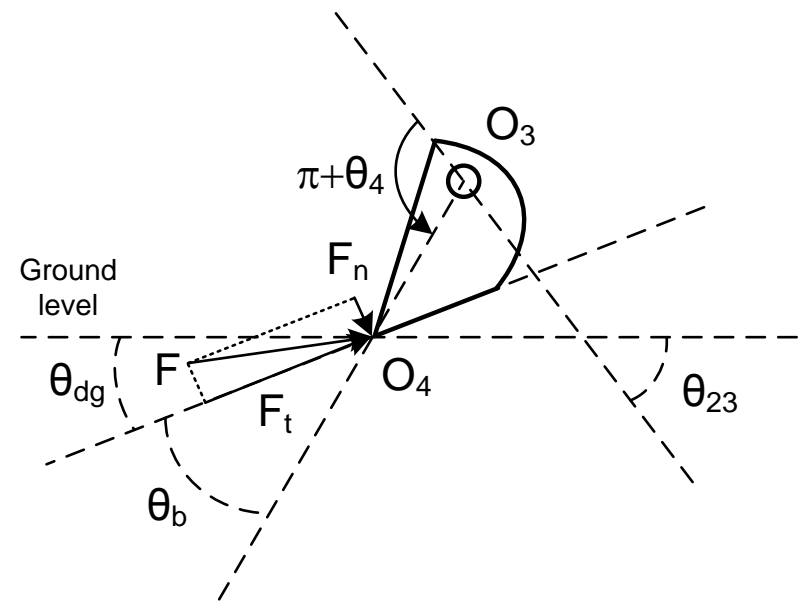

Figure 7: The interaction between the excavator bucket and the environment

According to Alekseeva et al. (1986), $F_{t}$ and $F_{n}$ are the tangential and normal components of the soil reaction force at the bucket, respectively. The tangential component can be calculated as

$$
F_{t}=k_{1} b h
$$

where $\mathrm{k}_{1}$ is the specific digging force in $\mathrm{N} / \mathrm{m}^{2}$, and $\mathrm{h}$ and $\mathrm{b}$ are the thickness and width of the cut slice of soil. The normal component $F_{n}$ is calculated as

$$
F_{n}=\Psi F_{t}
$$

where $\Psi=0.1-0.45$ is a dimensionless factor that depends on the digging angle, digging conditions, and the wear of the cutting edge.

So according to Figure 7, the loading torque is given as below:

$$
\tau_{L}=\left[\begin{array}{c}
\tau_{b} \\
a_{2}\left[F_{t} \sin \left(\theta_{2}-\theta_{d g}\right)-F_{n} \cos \left(\theta_{2}-\theta_{d g}\right)\right] \\
a_{3}\left[F_{t} \sin \left(\theta_{23}+\theta_{d g}\right)-F_{n} \cos \left(\theta_{23}+\theta_{d g}\right)\right] \\
-a_{4}\left(F_{t} \sin \theta_{b}+F_{n} \cos \theta_{b}\right)
\end{array}\right]
$$

Since during the digging operation, the joint variable $\theta_{1}$ is not changed. So, the elements $\mathrm{D}_{1 \mathrm{i}}, \mathrm{D}_{\mathrm{i} 1}, \mathrm{C}_{1 \mathrm{i}}, \mathrm{C}_{\mathrm{i} 1}, \Gamma_{1 \mathrm{i}}, \Gamma_{\mathrm{i} 1}(\mathrm{i}=1,2,3$, and 4$), \mathrm{G}_{1}, \mathrm{~B}_{\mathrm{ba}}, \tau_{1}$ and $\tau_{\mathrm{b}}$ are not specified here.

\section{$4 \quad$ INTELLIGENT CONTROL OF EXCAVATORS}

Usually, the excavator is always required to carry out tasks involving contact with its environment, such as levelling and digging. In moving towards autonomous excavation, it is necessary to develop the controller that is robust to uncertainties associated with such tasks.

Although there are some pronounced differences between the classical robot manipulator and the robotic excavation (Ha et al., 2002), but there are also some parallels. Therefore, there are many control approaches which have been developed for the robot manipulator that can be adopted by the robotic excavation. In this section, we will firstly review the conventional control approaches: computed torque and PID, and then introduce three control approaches: adaptive control, 
robust control, and iterative learning control which have been developed on the fully actuated robot manipulator.

\subsection{Computed torque control}

Using the dynamic model of excavators in (7), the conventional computed torque control law is given as below:

$$
U_{d}=\hat{D}\left(\theta_{d}\right) \dot{\theta}_{v}+\hat{C}\left(\theta_{d}, \dot{\theta}_{d}\right) \dot{\theta}_{d}+\hat{G}\left(\theta_{d}\right)+\hat{B}\left(\dot{\theta}_{d}\right)
$$

where $\dot{\theta}_{v}=\ddot{\theta}_{d}-k_{v} \dot{e}-k_{p} e, e=\theta-\theta_{d}$ is the tracking error, $\mathrm{k}_{\mathrm{v}}$ and $\mathrm{k}_{\mathrm{p}}$ are linear gains to be designed, $\hat{D}(\theta)$ is the estimated inertia; $\hat{C}(\theta, \dot{\theta})$ is the estimated Coriolis and centripetal effects; $\hat{G}(\theta)$ is the estimated gravity forces; $\hat{B}(\dot{\theta})$ is the estimated friction effects, $\mathrm{U}_{\mathrm{d}}$ is the desired torques applied to the system, $\theta_{d}, \dot{\theta}_{d}, \ddot{\theta}_{d}$ are the desired joint link angle, angular velocity, and angular acceleration, respectively.

It is found that the computed torque control approach is specified by the inverse dynamics of the excavator (7). The controller (17) generates the generalised torques to be applied to the excavator producing the desired motion under ideal condition. The simulation results of tracking a desired motion are presented in Figure 8, and the computed torques for the boom, stick, and bucket are presented in Figure 9, Figure 10, and Figure 11, respectively. The numerical parameters used in the simulation are given in Appendix.

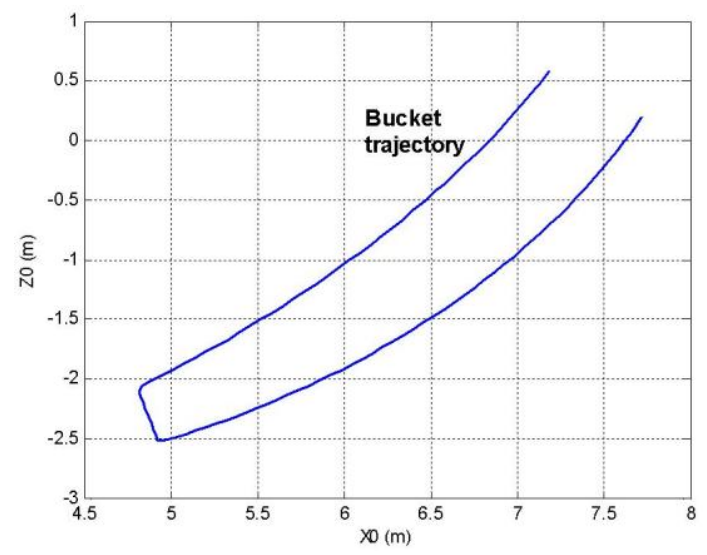

Figure 8: The bucket trajectory

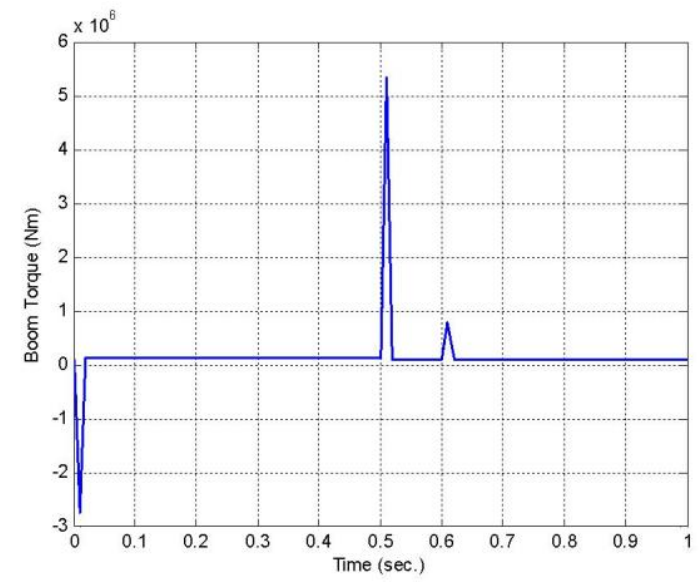

Figure 9: The boom torque

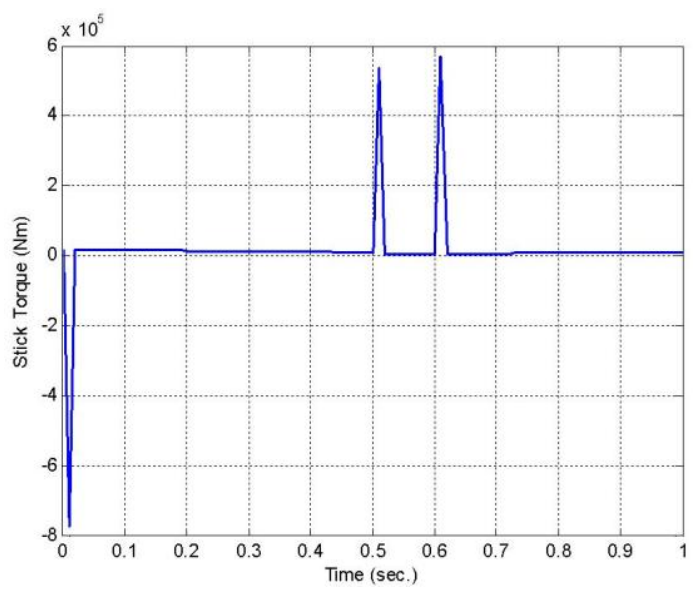

Figure 10: The stick torque

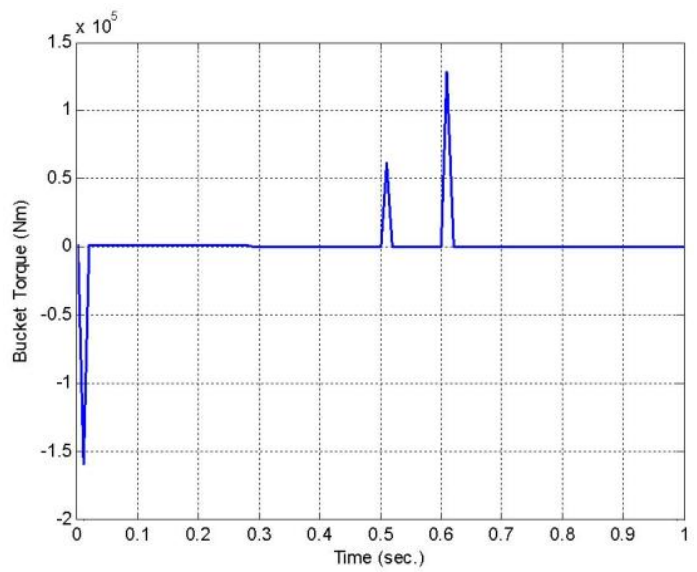

Figure 11: The bucket torque

\subsection{PD control}

Due to the uncertainties in real environment, only the computed torque control approach is not sufficient to control the motion of the excavator. So, according to Koivo et al. (1996), the PD controller is used as a secondary controller associated with the computed torque controller. The control architecture is presented in Figure 12.

Based on Figure 12, the PD controller is given as below:

$$
U_{P D}=K_{P} e(t)+K_{D} \dot{e}(t)+K_{f}\left[\tau_{L}(t)-\tau_{L}^{d}(t)\right]
$$

where $e(t)=\theta(t)-\theta_{d}(t), \tau_{L}^{d}(t)$ is the desired torque exerted by the bucket on the ground, and $\mathrm{K}_{\mathrm{P}}, \mathrm{K}_{\mathrm{D}}$ are the gains of the PD controller, $\mathrm{K}_{\mathrm{f}}$ is the proportional gain on the torque error.

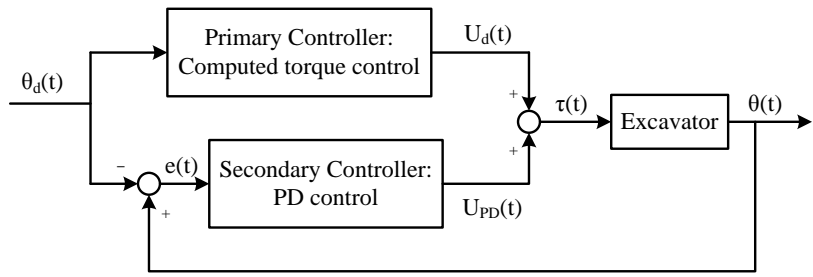

Figure 12: Control architecture 


\subsection{Adaptive control}

Due to the uncertainties in real environment, only the computed torque control approach is not sufficient to control the motion of the excavator. Therefore adaptive, robust and iterative learning is considered. Currently, there are many adaptive control approaches that have been developed on robot manipulators (Slotine et al. 1998, Johansson, 1990, Yu 1998). The main issues of adaptive control for robot manipulators are to adapt to uncertainties (e.g. uncertain parameters, payload changes, and unmodelled dynamics), and to avoid the use of the inverse of the estimated inertia matrix and the joint accelerations. So, these issues should be considered when developing adaptive control approaches for the excavator.

According to Theorem 2 in $\mathrm{Yu}$ (1998), the following adaptive control law is defined.

$$
U(t)=\tau_{n}(t)+\tau_{l}(t)
$$

where $\tau_{1}(\mathrm{t})$ is a linear feedback control part which is given in the following form,

$$
\tau_{l}=-\left(P_{l l}+P_{c c} \Gamma^{-1} P_{c c}\right) \dot{\tilde{q}}-P_{l l} P_{c c}^{-1} \Gamma \tilde{q}
$$

and $\tau_{\mathrm{n}}(\mathrm{t})$ is a nonlinear feedforward term which is given below,

$$
\tau_{n}=\hat{D}(q)(\dot{v}-\mu s)+\hat{C}(q, \dot{q}) v+\hat{G}(q)
$$

$\mathrm{P}_{1 l}$ is a symmetric positive definite matrix, $\mathrm{P}_{c c}=\mathrm{P}_{c c}{ }^{\mathrm{T}}, \Gamma=\Gamma^{\mathrm{T}}$, and $\mathrm{P}_{12}=\mathrm{P}_{\mathrm{cc}}{ }^{-1} \Gamma$ are the constant positive matrices, $\tilde{q}=q-q_{d}$ is the tracking error, and $v=\dot{q}_{d}-P_{12} \tilde{q}, s=\dot{\tilde{q}}+P_{12} \tilde{q}$.

Since

$$
W(t) \hat{\Theta}+W_{0}(t)=\hat{D}(q)(\dot{v}-\mu s)+\hat{C}(q, \dot{q}) v+\hat{G}(q)
$$

where $\widetilde{\Theta}=\hat{\Theta}-\Theta$, so, the updating law is chosen as

$$
\dot{\hat{\Theta}}=\dot{\widetilde{\Theta}}=-K_{d} W^{T}(t) s
$$

where $K_{d}$ is a symmetric positive definite matrix.

\subsection{Robust control}

Define the state errors as

$$
\tilde{x}=\left[\begin{array}{c}
\dot{q} \\
\tilde{q}
\end{array}\right]=\left[\begin{array}{l}
\dot{q}-\dot{q}_{d} \\
q-q_{d}
\end{array}\right]
$$

According to Yu et al. (1994), if the dynamic model (7) with constant but unknown parameters $\Theta$, the robust control law is given as below

$$
\begin{aligned}
& \bar{W}(t) \Theta(t)+\bar{W}_{0}(t) \\
& =D(q)\left\{\dot{v}-\mu\left(\dot{\tilde{q}}+P_{12} \tilde{q}\right)\right\}+C(q, \dot{q}) v+G(q) \\
& \mathrm{U}(\mathrm{t})=\mathrm{T}_{\mathrm{f}}(\mathrm{t})+\mathrm{T}_{1}(\mathrm{t}) \\
& \mathrm{T}_{1}(\mathrm{t})=-\left(\mathrm{P}_{11}+\mathrm{P}_{\mathrm{cc}} \Gamma^{-1} \mathrm{P}_{\mathrm{cc}}\right) \mathrm{P}_{1} \tilde{x}(t)+P_{c c} \tilde{q}(t) \\
& \mathrm{T}_{\mathrm{f}}(\mathrm{t})=\bar{W}(t) \Theta_{v}(t)+\bar{W}_{0}(t) \\
& \Theta_{v}(t)=-F_{1}(t) \bar{\Theta} \quad \bar{\theta}_{i} \geq \theta \forall i \\
& F_{1}(t)=\operatorname{diag}\left\{\operatorname{sgn}\left(f_{1}\right), \operatorname{sgn}\left(f_{2}\right), \ldots, \operatorname{sgn}\left(f_{p}\right)\right\} \\
& F(t)=\tilde{x}^{T} P_{1}^{T} \bar{W}(t)=\left[f_{1}(t), f_{2}(t), \ldots, f_{p}(t)\right]
\end{aligned}
$$

where $\Theta_{\mathrm{v}}(\mathrm{t})$ is a switching-function vector designed according to the robust control approach, and $\mathrm{P}_{1}=\left[\mathrm{I}_{\mathrm{nxn}} \mathrm{P}_{12}\right]$.

\subsection{Iterative learning control}

Iterative learning control is a method of tracking control for systems that work in a repetitive mode (Bristow et al., 2006). During each repetition, the system is required to perform the same action over and over again with high precision. By using information from previous repetitions, a suitable control action which is given as below can be found iteratively:

$$
U_{k+1}=U_{k}+K \cdot e_{k}
$$

where $\mathrm{U}_{\mathrm{k}}$ is the input to the system during the $\mathrm{k}^{\text {th }}$ repetition, $\mathrm{e}_{\mathrm{k}}=\theta_{\mathrm{d}}-\theta_{\mathrm{k}}$ is the tracking error during the $\mathrm{k}^{\mathrm{th}}$ repetition, and $\mathrm{K}$ is a design parameter. Based on (32), the iterative learning control scheme (Yu, et al., 2003) presented in Figure 13 can be designed for the excavator.

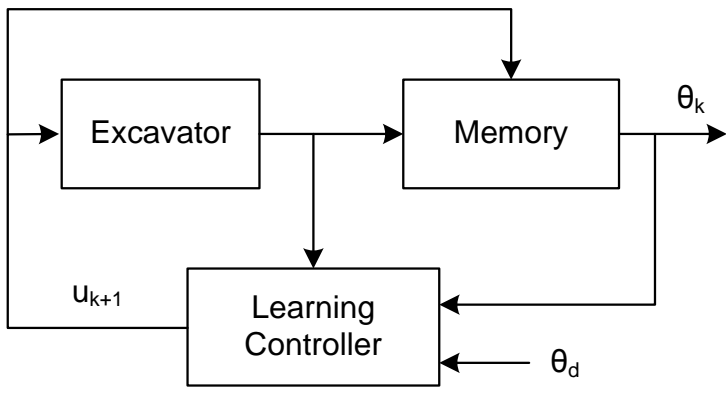

Figure 13: The iterative learning control scheme for the excavator

\section{REMOTE CONTROL OF EXCAVATORS}

Research on Wireless Networked Control Systems (WNCS) or wireless networks, e.g. MANET mostly relies on computer simulation studies since launching real experiments are expensive and time consuming (Kotz et al., 2004), (Conti and Giordano, 2007). Models for the plant, controller and the network can be simulated using a mathematical simulation package e.g. MATLAB-SIMULINK, or network simulators e.g. Optimised Network Engineering Tool (OPNET) (Chang, 1999), Network Simulator version 2 (NS2) (Fall and Varadhan, 2006). MATLAB-SIMULINK is well accepted in the research community as a powerful tool for modelling systems and implementing control algorithms. However, it has limitations in simulating computer networks. Toolboxes for MATLAB e.g., TrueTime (Cervin et al., 2003), (Cervin et al., 2002), (Cervin et al., 2007), (Henriksson et al., 2002), (Eker and Cervin, 1999) from the Lund institute in Sweden, allow wireless networked control systems simulation. However, they do NOT have the flexibility to set many vital MANET parameters e.g. node movement model, wireless signal propagation model etc. On the other hand, OPNET is an advanced simulation package that allows detailed communication network simulation (Chang, 1999). Many aspects of the network such as network type and technology, network data rate, node movement, wireless signal propagation model etc. can be specified in OPNET. However, it is a tedious task to implement dynamic system models and control algorithms using the Proto-C language of OPNET. Therefore, a co-simulation framework utilising MATLAB-SIMULINK to model the plant-controller and 
OPNET to simulate the network has been developed as shown in Figure 14 to accelerate the WNCS research by producing more realistic simulation results (Hasan et al., 2009), (Hasan et al., 2008), (Hasan et al., 2007).

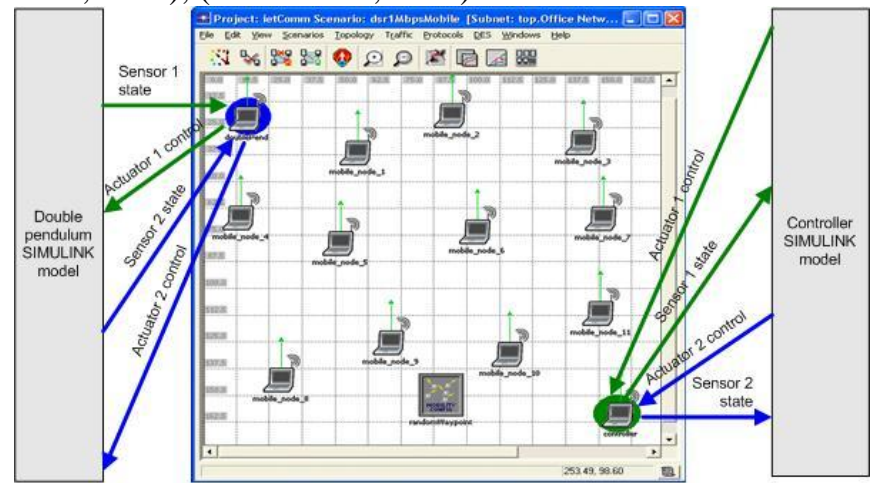

Figure 14: Interactive SIMULINK-OPNET co-simulation.

The system can be launched in diverse situations e.g. indoor (nuclear plant), outdoor (in a desert for military operation). Therefore, the terrain and the environment will affect the excavator movement and the wireless communication performance, respectively. Depending on the terrain, either wheeled (JCB, 2009b) or tracked (JCB, 2009a) excavator can be used.

The modelling or simulation results might vary depending on the environment of the wireless network, e.g., office area, open field etc. (Kotz et al., 2004), (Liu et al., 2004) and efforts should be focused on more realistic settings (Tschudin et al., 2005). Therefore, the co-simulation framework should allow the researchers to specify the environment where the investigation is being carried out.

Real radio signals are more complicated than the simple models used in many simulation studies. Simpler propagation models can assume symmetric wireless links, independence from ground height etc. that might produce impractical results. Moreover, the characteristics of the radio signal also change depending on the environment, e.g., indoor, outdoor etc. It can be noted that wireless networks have a smaller transmission range in the office than in an open field because of less interference from other electrical equipments, walls etc. Furthermore, simulation of the same network in different simulation packages might produce different results. This can be explained by the physical layer considered in the simulation package (Conti and Giordano, 2007). In case of WNCS over MANET, the environment model, i.e., experiment area, number of nodes, movement model of the nodes etc. are major design issues.

A comparison between computer simulation and real world wireless network experiments can be found in (Kotz et al., 2004), (Liu et al., 2004), (Newport, 2004) . The three radio signal propagation models have been investigated in (Kotz et al., 2004) as shown in Figure 15. Model 1 involves two components: path loss and fading. Model 2 is the two-ray-ground reflection model that uses only the path loss component. Finally, model 3 represents the ideal propagation model. The comparison, shown in Figure 15, revealed that model 1 exhibits the closest behaviour to the real world experiment (Kotz et al., 2004).

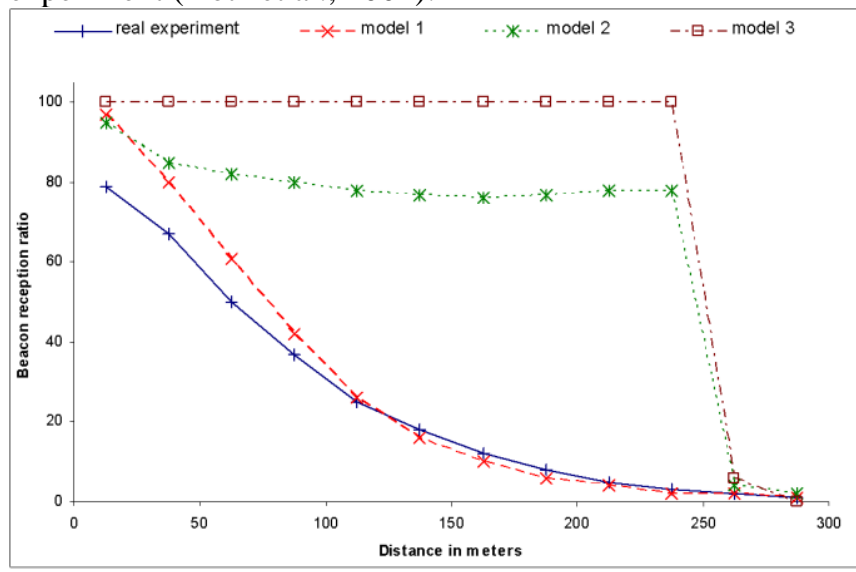

Figure 15: Comparison of three simulation models with real world experiment (Kotz et al., 2004).

Many WNCS researches e.g. (Ploplys et al., 2004), (Ploplys, 2003), (Colandairaj et al., 2005), (Colandairaj et al., 2007), (Colandairaj et al., 2006), (Andersson et al., 2005), (Willig et al., 2002), (Walsh and Ye, 2001) are based on mainly IEEE 802.11 standards. Various versions of the IEEE 802.11 standards, namely a, b, g, e, carry the difference at the physical layer. They utilise the free Industrial Scientific Medical (ISM) $2.4 \mathrm{GHz}$ band and support data rates $1,2,11,54 \mathrm{Mbps}$. IEEE 802.11 defines pure ad-hoc mode as Distributed Coordination Function (DCF) and uses Carrier Sense Multiple Access with Collision Avoidance (CSMA/CA) as the Medium Access Control (MAC) protocol (Pellegrini et al., 2006). The MAC protocol determines how the nodes access the shared network medium and it is responsible for satisfying the time-critical requirements (Lian et al., 2001).

Implementing control networks using IEEE 802.11 standards will allow low-cost and easily deployable WNCS. Unfortunately, CSMA networks are generally considered nondeterministic. However, if the network protocol supports prioritising of messages, then high priority messages will have higher chance of timely transmission and collision avoidance (Zhang et al., 2001), (Ploplys et al., 2004). Contention based protocols e.g. CSMA are not appropriate for real time communication as they require handshaking which increases the delay (Akyildiz and Kasimoglu, 2004).

Wireless networks inherently suffer from security problems as signals are broadcast to all receivers. Two types of security issues can be identified: signal integrity and authentication. The concern of signal integrity comes from the interference from other radio transmitters e.g. microwave ovens, cordless phones etc. (Ploplys et al., 2004). This problem can be crucial for IEEE 802.11 as it uses the ISM $2.4 \mathrm{GHz}$ band. However, the spread spectrum techniques implemented by the standards can mitigate the interference in most cases (Pellegrini et al., 2006). The IEEE 802.11 standard offers a (Wireless Local Area Network (WLAN) authentication mechanism called Wired Equivalent Privacy (WEP) from the MAC layer. 
However, the security provided is not adequate (Pellegrini et al., 2006).

TCP/IP is not appropriate for MANET as it uses connection oriented packet transfer (Conti and Giordano, 2007). For real-time applications, the UDP protocol is shown more suitable than the TCP in terms of delay and delay jitter (Liu et al., 2002) as shown in Figure 16. Retransmission for TCP will simply produce unwanted network traffic as WNCS carries real time data. On the other hand, UDP offers low overheads as it does not maintain connections and discards obsolete lost packets by avoiding retransmissions. Therefore, it is preferable for networked control applications (Ploplys et al., 2004). UDP is also often chosen to validate the simulation results for wireless networks (Liu et al., 2004).

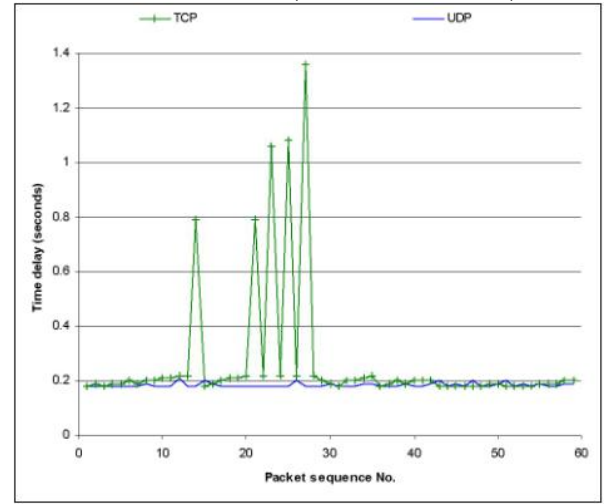

Figure 16: Comparison of delay and jitter between TCP and UDP (Liu et al., 2002), (Liu et al., 2005).

\section{CONCLUSIONS AND FUTURE WORK}

The paper has reported the work conducted in an on-going project. The key issues in remotely controllable excavators have been identified. An overall architecture has been proposed and functions of each block of the architecture have been discussed. Some simulation work has been conducted to demonstrate the proposed system.

We will conduct the further simulation on the whole system. The experimental study will be investigated as well.

\section{APPENDIX}

Some symbol definitions used in section 3:

$\mathrm{M}_{\mathrm{bo}}=1566 \mathrm{~kg}$ : mass of boom

$\mathrm{M}_{\mathrm{st}}=735 \mathrm{~kg}$ : mass of stick

$\mathrm{M}_{\mathrm{bu}}=432 \mathrm{~kg}$ : mass of bucket

$\mathrm{I}_{\mathrm{bo}}=14250.6 \mathrm{~kg} \cdot \mathrm{m}^{2}:$ moment of inertia of boom

$\mathrm{I}_{\mathrm{st}}=727.7 \mathrm{~kg} \cdot \mathrm{m}^{2}:$ moment of inertia of stick

$\mathrm{I}_{\mathrm{bu}}=224.6 \mathrm{~kg} \cdot \mathrm{m}^{2}:$ moment of inertia of bucket

$\theta_{1}$ : angle of base

$\theta_{2}$ : angle of boom

$\theta_{3}$ : angle of stick

$\theta_{4}$ : angle of bucket $\theta_{\mathrm{b}}$ : angle between bucket bottom and $\mathrm{X}_{4}$-axis

$\theta_{\mathrm{dg}}$ : angle between bucket edge and horizontal line

$\mathrm{a}_{1}=0.05 \mathrm{~m}: \mathrm{O}_{0} \mathrm{O}_{1}$

$\mathrm{a}_{2}=5.16 \mathrm{~m}: \mathrm{O}_{1} \mathrm{O}_{2}$

$\mathrm{a}_{3}=2.59 \mathrm{m:} \mathrm{O}_{2} \mathrm{O}_{3}$

$\mathrm{a}_{4}=1.33 \mathrm{m:} \mathrm{O}_{3} \mathrm{O}_{4}$

$\mathrm{r}_{2}=2.71 \mathrm{m:} \mathrm{O}_{1} \mathrm{G}_{2}$

$\mathrm{r}_{3}=0.64 \mathrm{m:} \mathrm{O}_{2} \mathrm{G}_{3}$

$\mathrm{r}_{4}=0.65 \mathrm{m:} \mathrm{O}_{3} \mathrm{G}_{4}$

$\alpha_{2}=0.2566 \mathrm{rad}: \angle \mathrm{G}_{2} \mathrm{O}_{1} \mathrm{O}_{2}$

$\alpha_{3}=0.3316 \mathrm{rad}: \angle \mathrm{G}_{3} \mathrm{O}_{2} \mathrm{O}_{3}$

$\alpha_{4}=0.3944 \mathrm{rad}: \angle \mathrm{G}_{4} \mathrm{O}_{3} \mathrm{O}_{4}$

$\mathrm{B}_{\mathrm{bo}}$ : viscous friction coefficient of boom

$\mathrm{B}_{\mathrm{st}}:$ viscous friction coefficient of stick

$\mathrm{B}_{\mathrm{bu}}$ : viscous friction coefficient of bucket

$\mathrm{g}=9.81 \mathrm{~N} / \mathrm{kg}$ : acceleration due to gravity

$\mathrm{T}_{\mathrm{s}}=10 \mathrm{~ms}$ : sampling time

\section{ACKNOWLEDGEMENT}

The authors would like to thank Mr Sam Wane for the useful discussion and JCB, Sellafield for initiating this work.

\section{REFERENCES}

Akyildiz, I. F. and Kasimoglu, I. H. (2004) Wireless sensor and actor networks: research challenges. Ad Hoc Networks Journal (Elsevier), vol. 2, no. 4, pp. 351-367.

Alekseeva, T. V., Artem'ev, K. A., Bromberg, A. A. and Voitsekhovskii, R. L. (1986) Machines for earthmoving work: theory and calculations, Rotterdam: A. A. Balkema.

Andersson, M., Henriksson, D., Cervin, A. and Årzén, K. E. (2005) 'Simulation of Wireless Networked Control Systems', Proc. of the 44th IEEE Conference on Decision and Control and European Control Conference (ECC), pp. 476-481.

Bernold, L. E. (1993) 'Motion and path control for robotic excavation', J. Aerosp. Eng., vol. 6, no. 1, pp. 1-18.

Bradley, D. V. and Seward, D. W. (1998) 'The development, control and operation of an autonomous robotic excavator', $J$. Intelligent Robot. Syst., vol. 21, pp. 73-97.

Bristow, D. A., Tharayil, M. and Alleyne, A. G. (2006) 'A survey of iterative learning control', IEEE Control Systems Magazine, vol. 26, Issue 3, June, pp. 96-114.

Cervin, A. 2003, Integrated Control and Real time Scheduling, $\mathrm{PhD}$ thesis, Lund Institute of Technology.

Cervin, A., Hanriksson, D., Lincoln, B., Eker, J. and Årzén, K. E. (2003) How does control timing affect performance? Analysis and Simulation of Timing using Jitterbug and TrueTime. IEEE Control Systems Magazine, vol. 23, no. 3, pp. 16-30.

Cervin, A., Henriksson, D., Lincoln, B., Eker, J. and Årzén, K. E. (2002) 'Jitterbug and TrueTime: Analysis Tools for Real-Time Control Systems', Proc. of the 2nd Workshop on Real-Time Tools, Copenhagen, Denmark.

Cervin, A., Ohlin, M. and Henriksson, D. (2007) 'Simulation of Networked Control Systems Using TrueTime', Proc. of the 3rd International Workshop on Networked Control Systems: Tolerant to Faults, Nancy, France. 
Chang, X. (1999) 'Network simulations with OPNET', Proc. of the 31 st conference on Winter simulation: Simulation - a bridge to the future, Arizona, USA, pp. 307-314.

Colandairaj, J., Irwin, G. W. and Scanlon, W. G. (2005) 'Analysis and Co-Simulation of an IEEE 802.11B Wireless Networked Control System', Proc. of the 16th IFAC World Congress, Prague, Czech Republic.

Colandairaj, J., Irwin, G. W. and Scanlon, W. G. (2006) 'An integrated approach to wireless feedback control', Proc. of the UKACC International Control Conference, Glasgow, UK.

Colandairaj, J., Irwin, G. W. and Scanlon, W. G. (2007) 'A Co-Design Solution for Wireless Feedback Control', Proc. of the IEEE International Conference on Networking, Sensing and Control, London, UK, pp. 404-409.

Conti, M. and Giordano, S. (2007) Multihop Ad Hoc Networking: The Theory. IEEE Communications Magazine, vol. 45, no. 4, pp. 76-86.

Dimaio, S. P., Salcudean, S. E., Reboulet, C., Tafazoli, S., and Zaad, K. H. (1998) 'A virtual excavator for controller development and evaluation', Proc. of the IEEE Int. Conf. on Robotics and Automation, May.

Eker, J. and Cervin, A. (1999) 'A Matlab Toolbox for Real-Time and Control Systems Co-Design', Proc. of the 6th International Conference on Real-Time Computing Systems and Applications, Hong Kong, P.R. China, pp. 320-327.

Fua, P. (1993) 'A parallel stereo algorithm that produces dense depth maps and preserves image features', in Machine Vision and Application. New York: Springer-Verlag.

Fall, K. and Varadhan, K., (2006) The Ns Manual (formerly ns Notes and Documentation), The VINT project [online]. Available from: <http://www.isi.edu/nsnam/ns/doc/ns_doc.pdf > [Accessd: Mar 2006]

Hasan, M. S., Yu, H., Carrington, A. and Yang, T. C. (2009) Co-simulation of wireless networked control systems over mobile ad hoc network using SIMULINK and OPNET. the Journal of IET Communications (accepted for publication).

Hasan, M. S., Yu, H., Griffiths, A. and Yang, T. C. (2007) 'Interactive co-simulation of MATLAB and OPNET for Networked Control Systems', Proc. of the 13th International Conference on Automation and Computing, Stafford, UK, pp. 237-242.

Hasan, M. S., Yu, H., Griffiths, A. and Yang, T. C. (2008) 'Co-simulation framework for Networked Control Systems over multi-hop mobile ad-hoc networks', Proc. of the 17th IFAC World Congress, the International Federation of Automatic Control, Seoul, Korea, pp. 12552-12557.

Ha, Q. P., Nguyen, Q. H., Rye, D. C., and Durrant-Whyte, H. F. (2000) 'Impedance control of a hydraulically-actuated robotic excavator', Automat. Construction. vol. 9, no. 5, pp. 421-435.

Ha, Q., Santos, M., Nguyen, Q., Rye, D., and Durrant-Whyte, H. (2002) 'Robotic excavation in construction automation', IEEE Robotics \& Automation Magazine, vol. 9, issue 1, March, pp. 20-28.

Heinrichs, B., Sepehri, N., and Thornton-Trump, A. B. (1997) 'Position-based impedance control of an industrial hydraulic manipulator', IEEE Contr. Syst. Mag., vol. 17, February, pp.46-52.

Henriksson, D., Cervin, A. and Årzén, K. E. (2002) 'TrueTime: Simulation of Control Loops Under Shared Computer Resources', Proc. of the 15th IFAC World Congress on Automatic Control, Barcelona, Spain.

JCB, (2009a) Tracked excavators [online]. Available from: <http://www.jcb.com/products/MachineOverview.aspx?RID=2 $>$ [Accessd: 06 May 2009].
JCB, (2009b) Wheeled excavators [online]. Available from: <http://www.jcb.com/products/MachineOverview.aspx?RID=3 $\geq$.

Johansson, R. (1990) 'Adaptive control of robot manipulator motion', IEEE Trans. on Robotics and Automation, vol. 6, pp. 483-490.

Kim, D., Oh, K. W., Hong, D., Park, J., and Hong, S. (2008) 'Remote control of excavator with designed haptic device', Proc. of Int. Conf. on Control, Automation and Systems, Seoul, Korea, October.

Koivo, A. J. (1994) 'Kinematics of excavators (backhoes) for transferring surface material', J. Aerosp. Eng., vol. 7, no. 1, pp. 17-32.

Koivo, A. J., Thoma, M., Kocaoglan, E., and Andrade-Cetto, J. (1996) 'Modelling and control of excavator dynamics during digging operation', J. Aerosp. Eng., vol. 9, no. 1, January, pp. 10-18.

Kotz, D., Newport, C., Gray, R. S., Liu, J., Yuan, Y. and Elliott, C. (2004) 'Experimental Evaluation of Wireless Simulation Assumptions', Proc. of the ACM/IEEE International Symposium on Modelling, Analysis and Simulation of Wireless and Mobile Systems (MSWiM), pp. 78-82.

Kuc, T., Nam, K. and Lee, J. S. (1991) 'An iterative learning control of robot manipulators', IEEE Trans. on Robotics and Automation, vol. 7, no. 6, December, pp. 835-842.

Lawrence, P. D., Salcudean, S. E., Sepehri, N., Chan, D., Bachmann, S., Parker, N., Zhu, M., and Frenette, R. (1995) 'Coordinated and force-feedback control of hydraulic excavators', Proc. of the $4^{\text {th }}$ Int. Symp. on Experimental Robotics, Stanford, California, June.

Le, A. T., Nguyen, Q. H., Ha, Q. P., Rye, D. C., Durrant-Whyte, H. F., Stevens, M., and Boget, V. (1998) 'Towards autonomous excavation', Field and Service Robotics, A. Zelinsky, Ed. New York: Springer-Verlag.

Lian, F. L., Moyne, J. R. and Tilbury, D. M. (2001) Performance Evaluation of Control Networks: Ethernet, ControlNet and DeviceNet. IEEE Control Systems Magazine, vol. 21, no. 1, pp. 66-83.

Liu, J., Yuan, Y., Nicol, D. M., Gray, R. S., Newport, C. C., Kotz, D. F. and Perrone, L. F. (2004) 'Simulation validation using direct execution of wireless ad-hoc routing protocols', Proc. of the 18th Workshop on Parallel and Distributed Simulation (PADS'04), Austria, pp. 7-16.

Liu, P. X., Meng, M., Ye, X. and Gu, J. (2002) 'An UDP-Based Protocol for Internet Robots', Proc. of the 4th World Congress on Intelligent Control and Automation, China, pp. 59-65.

Liu, P. X., Meng, M. Q. H., Liu, P. R. and Yang, S. X. (2005) An End-to-End Transmission Architecture for the Remote Control of Robots Over IP Networks. IEEE/ASME Transactions on Mechatronics, vol. 10, no. 5, pp. 560-570.

$\mathrm{Lu}, \mathrm{Z}$. and Goldenberg, A. A. (1995) 'Robust impedance control and force regulation: theory and experiment', Int. J. Robotic. Res., vol. 14, no. 3, pp. 225-254.

Makkonen, T., Nevala, K. and Heikkila, R. (2006) 'A 3D model based control of an excavator', Automation in Construction, vol. 15, pp. 571-577.

Merritt, H. E. (1967) Hydraulic control systems, New York: Wiley.

Newport, C. (2004) Simulating mobile ad hoc networks: a quantitative evaluation of common MANET simulation models. Dartmouth College Science, Available from: $\langle$ http://cmc.cs.dartmouth.edu/cmc/papers/newport:thesis.pdf $\rangle$.

Pellegrini, F. D., Miorandi, D., Vitturi, S. and Zanella, A. (2006) On the Use of Wireless Networks at Low Level of Factory Automation Systems. IEEE Transactions on Industrial Informatics, vol. 2, no. 2, pp. 129-143. 
Parker, N. R., Salcudean, S. E., and Lawrence, P. D. (1993) 'Application of force feedback to heavy duty hydraulic machines', Proc. IEEE Conf. on Robotics and Automation, Atlanta, USA, May.

Ploplys, N. J. 2003, Wireless feedback control of mechanical systems, MSc thesis, University of Illinois.

Ploplys, N. J., Kawka, P. A. and Alleyne, A. G. (2004) Closed-loop Control over Wireless Networks. IEEE Control Systems Magazine, vol. 24, no. 3, pp. 58-71.

Roover, D. and Bosgra, O. H. (2000) 'Synthesis of robust multivariable iterative learning controller with application to a wafer stage motion system', Int. J. Contr., vol. 73, no. 10, pp. 968-979.

Saeedi, P., Lawrence, P. and Lowe, D. (2000) '3-D motion tracking of a mobile robot in a natural environment', Proc. of IEEE Int. Conf. Robotics and Automation, 2000, pp.1682-1687.

Saeedi, P., Lawrence, P. D., Lowe, D. G., Jacobsen. P., Kusalovic, D., Ardron, K., and Sorensen, P. H. (2005) 'An autonomous excavator with vision-based track-slippage control', IEEE Trans. on Control Systems Technology, vol. 13, no. 1, January, pp. 67-84.

Sakaida, Y., Chugo, D., Yamamoto, H., and Asama, H. (2008) 'The analysis of excavator operation by skilful operator', SICE Annual Conf. Japan, August.

Schmid, R. M. C. and Bauckhage, C. (1998) 'Comparing and evaluating interest points', Proc. $6^{\text {th }}$ Int. Conf. Computer Vision, 1998, pp.230-235.

Shapiro, L., Zisserman, A., and Brady, M. (1995) '3-D motion recovery via affine epipolar geometry’, Int. J. Comput. Vis., vol. 16, pp. 147-182.

Singh, S. (1995) 'Synthesis of tactical plans for robotic excavation', PhD dissertation, Robot. Inst., Carnegie Mellon Univ., Pittsburgh, PA, USA.

Slotine, J. J. E. and Li, W. (1998) 'Adaptive manipulator control: a case study', IEEE Trans, on Automatic Control, vol. 33, pp. 995-1003.

Tafazoli, S. (1997) 'Identification of frictional effects and structural dynamics for improved control of hydraulic manipulators', $P h D$ dissertation, Dept. Elect. Comput. Eng., Univ. British Columbia, Vancouver, B. C., Canada.

Tafazoli, S., Lawrence, P. D., and Salcudean, S. E. (1999) 'Identification of inertial and friction parameters for excavator arms', IEEE Trans. on Robotics and Automation, vol. 15, no. 5, October, pp. 966-971.

Tafazoli, S., Salcudean, S. E., Hashtrudi-Zaad, K., and Lawrence, P. D. (2002) 'Impedance control of a teleoperated excavator', IEEE Trans. on Control Systems Technology, vol. 10, no. 3, May, pp. 355-367.

Tan, C. P., Zweiri, Y. H., Althoefer, K., and Seneviratne, L. D. (2005) 'Online soil parameter estimation scheme based on
Newton-raphson method for autonomous excavation', IEEE/ASME Trans. on Mechatronics, vol. 10, no. 2, April, pp. 221-229.

Tao, N., Zhao, D., Yamada, H., and Shui, N. (2008) 'A low-cost solution for excavator simulation with realistic visual effect', Proc. of the IEEE Conf. on Robotics, Automation and Mechatronics, September.

Tucker, R. L. (1999) 'Construction automation in the USA', Proc. $16^{\text {th }}$ Int. Symp. on Automat. Robotics Construction, Madrid, Spain.

Tschudin, C., Gunningberg, P., Lundgren, H. and Nordstrom, E. (2005) Lessons from experimental MANET research. Ad Hoc Networks Journal (Elsevier), vol. 3, no. 2, pp. 221-233.

Vaha, P. K. and Skibniewski, M. J. (1993) 'Dynamic model of excavator', J. Aerosp. Eng., vol. 6, April, pp. 148-158.

Vossoughi, G. and Salcudean, S. E. (2000) 'On the nonlinear control of hydraulic servo-systems', Proc. IEEE Int. Conf. Robot. Automat., San Francisco, CA. April, pp. 1276-1282.

Walsh, G. C. and Ye, H. (2001) Scheduling of Networked Control Systems. IEEE Control Systems Magazine, vol. 21, no. 1, pp. 57-65.

Willig, A., Kubisch, M., Hoene, C. and Wolisz, A. (2002) Measurements of a Wireless Link in an Industrial Environment Using an IEEE 802.11-Compliant Physical Layer. IEEE Transactions on Industrial Electronics, vol. 49, no. 6, pp. 1265-1282.

Yang, S., Jin, S., and Kwon, S. (2008) 'Remote control system of industrial field robot', Proc. of the IEEE Int. Conf. on Industrial Informatics, Daejeon, Korea, July.

Yu, H., Seneviratne, L. D. and Earles, S. W. E. (1994) 'Exponentially stable robust control law for robot manipulators', IEE Proc. Control Theory Application, vol. 141, no. 6, November, pp. 389-395.

$\mathrm{Yu}, \mathrm{H}$. (1998) 'Robust combined adaptive and variable structure adaptive control of robot manipulators', Robotica, vol. 16, pp. 623-650.

Yu, H., Yang, T. C. and Rigas, D. (2003) 'On iterative learning control', Proc. of the $22^{\text {nd }}$ Chinese Control Conference, Yichang, China, 11-14 August.

Zhang, W., Branicky, M. S. and Phillips, S. M. (2001) Stability of Networked Control Systems. IEEE Control Systems Magazine, vol. 21, no. 1, pp. 84-99.

Zweiri, Y. H., Seneviratne, L. D., and Althoefer, K. (2004) 'Parameter estimation for excavator arm using generalized Newton method', IEEE Trans. on Robotics, vol. 20, no. 4, August, pp. 762-767. 